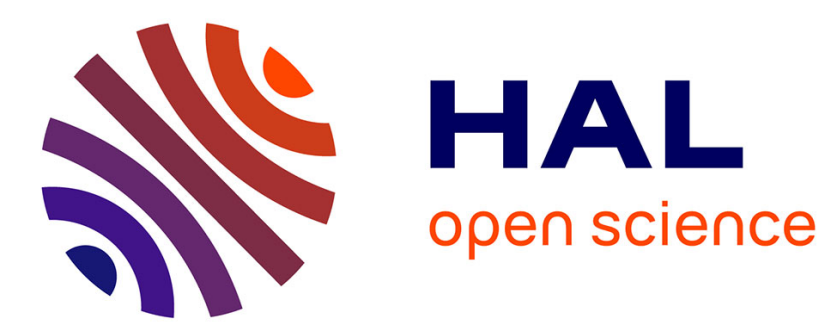

\title{
Études sur les accélérateurs de particules supraconducteurs
}

J. Kervizic

\section{To cite this version:}

J. Kervizic. Études sur les accélérateurs de particules supraconducteurs. Revue de Physique Appliquée, 1969, 4 (2), pp.188-189. 10.1051/rphysap:0196900402018801 . jpa-00243211

\section{HAL Id: jpa-00243211 https://hal.science/jpa-00243211}

Submitted on 1 Jan 1969

HAL is a multi-disciplinary open access archive for the deposit and dissemination of scientific research documents, whether they are published or not. The documents may come from teaching and research institutions in France or abroad, or from public or private research centers.
L'archive ouverte pluridisciplinaire HAL, est destinée au dépôt et à la diffusion de documents scientifiques de niveau recherche, publiés ou non, émanant des établissements d'enseignement et de recherche français ou étrangers, des laboratoires publics ou privés. 


\title{
ÉTUDES SUR LES AGCÉLÉRATEURS DE PARTICULES SUPRAGONDUGTEURS
}

\author{
J. KERVIZIC, \\ C.S.F., Domaine de Corbeville, 9I-Orsay.
}

\begin{abstract}
Résumé. - Cette communication expose sommairement des résultats d'étude sur les cavités HF supraconductrices, envisagées du point de vue de leurs applications aux accélérateurs de particules. L'étude d'un dispositif d'asservissement en phase du champ accélérateur est présentée, et nous donnons les grandes lignes d'un projet d'accélérateur linéaire pour électrons.

Abstract. - This paper presents some results on superconducting RF cavities, as a first step in their application to particle accelerators. The study under way concerns a phaselocked accelerating field device. The main features for an electron linear accelerator are also given.
\end{abstract}

Le travail, engagé à la Division Accélérateurs de la Compagnie générale de T.S.F., avec l'aide et les encouragements de l'Institut de Recherches Nucléaires de Strasbourg-Cronenbourg, s'est développé suivant deux plans. Expérimentalement, aux hyperfréquences, plusieurs modèles de cavités résonnantes ont été réalisés et étudiés, un dispositif de correction en fréquence a été mis au point, et des études sont actuellement en cours pour réaliser la stabilisation en phase du champ accélérateur. D'autre part, nous avons préparé l'élaboration d'un projet d'accélérateur linéaire pour électrons, en collaboration avec M. A. Septier. L'étude expérimentale a porté sur des cavités en cuivre au mode TM 010, à la fréquence de $1300 \mathrm{MHz}$. Ces cavités sont plombées intérieurement par électrolyse (le plombage est réalisé par les services compétents de la C.S.F.)

La surtension des cavités a été étudiée suivant la méthode de Septier et Viet, par accrochage sur un amplificateur (tube à ondes progressives) et examen des constantes du temps du signal HF. Les meilleurs résultats s'élèvent à $70 \times 10^{6}$ à $4,2 \circ \mathrm{K}$, et $110 \times 10^{6}$ à $2 \circ \mathrm{K}$, sous une puissance de quelques watts. Pour certaines expériences, une légère détérioration des facteurs de surtension a pu être constatée en cours d'étude; elle est due vraisemblablement à la présence de microfuites.

L'isolement thermique est rendu particulièrement efficace sur nos montages, une provision de vingt litres d'hélium liquide permettant en effet l'expérimentation pendant trois journées consécutives.

Un dispositif mécanique de correction en fréquence a été utilisé, sans qu'il conduise à une détérioration notable du facteur de surtension. Il s'agit d'un piston supraconducteur traversant la paroi de la cavité perpendiculairement à son axe et dans le plan médian. Il est commandé par vis micrométrique, ce qui lui confère une définition de $10 \mathrm{~Hz}$ environ, pour une amplitude totale de $400 \mathrm{kHz}$.

L'étude expérimentale de la stabilité en phase du champ nous a fourni des résultats assez contingents. 
Nous avons cru pouvoir en conclure à la nécessité de stabiliser ce champ en vue d'un usage accélérateur éventuel.

L'investigation théorique des différentes méthodes utilisables a conduit à nous engager vers la réalisation d'un asservissement en phase, dont l'étude est actuellement poursuivie par M. Hurt. Il s'agit de commander

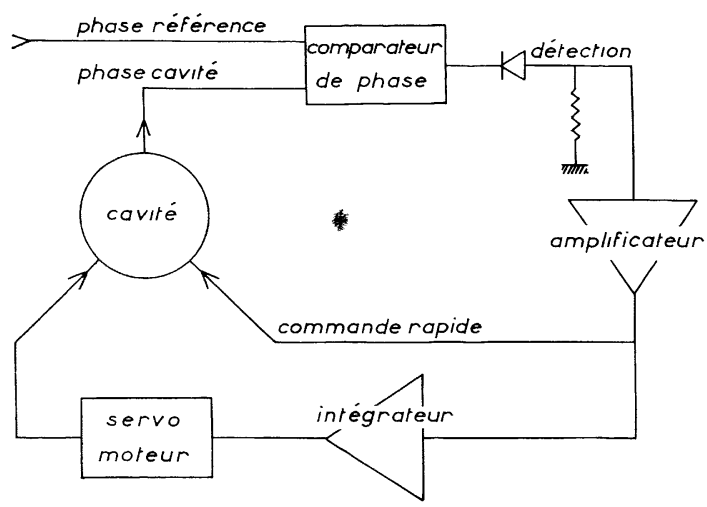

FIG. 1. - Circuit d'asservissement en phase. la polarisation d'un élément ferrimagnétique à partir d'une mesure d'erreur en phase (fig. 1). L'affaiblissement du facteur de surtension est alors directement proportionnel à l'amplitude des fluctuations à corriger. Il devrait pouvoir être maintenu dans des limites tolérables par l'usage simultané d'un système à réponse lente, mais n'ajoutant pas d'amortissement. De toute façon, les dissipations supplémentaires dues à la présence de l'élément rapide de correction se font à $300 \mathrm{oK}$, et sont donc de peu d'importance pour le bilan énergétique d'une éventuelle cavité accélératrice. La bande passante de l'asservissement devrait être de $10 \mathrm{kHz}$ en signal faible.

Nous avons concrétisé nos études par la préparation d'un projet d'accélérateur linéaire supraconducteur pour électrons. Des impératifs de simplicité nous ont conduits à envisager une seule section accélératrice à ondes stationnaires pouvant fournir quelques $\mathrm{MeV}$. La structure HF est en cuivre plombé, sa configuration est bi-périodique et elle devrait pouvoir fonctionner en impulsions longues. Son étude devrait s'effectuer avec la collaboration de l'équipe de l'Institut d'Électronique d'Orsay sous la direction de M. A. Septier. 